ISSN: 0213-2060

DOI: https://doi.org/10.14201/shhme2019371139164

\title{
EL GRAN INCENDIO CASTELLANO DE 949. HUELLA DIPLOMÁTICA Y MEMORIA HISTÓRICA DE UN DESASTRE NATURAL $^{1}$
}

\author{
The Great Castilian Wildfire of 949. Diplomatic Traces and Historical Memory \\ of a Natural Disaster
}

David PETERSON

Depto. de Historia, Geografía y Comunicación. Facultad de Humanidades y Comunicación. Universidad de Burgos. Paseo de Comendadores, s/n. E-09001 BURGOS. C. e.: dpeterson@ubu.es

Recibido: 2017-09-22

Revisado: 2018-04-04

Aceptado: 2019-04-30

RESUMEN: Un gran incendio a mediados del siglo x asoló la Meseta Norte, con especial incidencia en Castilla, dejando su huella no solo en las fuentes narrativas del momento y de generaciones posteriores sino también, de manera excepcional, en la diplomática, donde se observa un pico extraordinario de actividad en los meses posteriores al desastre. Se aprecia de manera especialmente nítida en un incomprendido dosier de doce documentos, datados en el primer semestre de 950, que se refieren al monasterio de Buezo en la Bureba, pero que están insertos en el Becerro Gótico de Valpuesta. Esta inhabitual coincidencia entre un hecho narrado en la analística y su huella en la diplomática ha pasado desapercibida hasta ahora, pero nos ofrece una perspectiva singular sobre el funcionamiento del campesinado en momentos de crisis y la relación entre tal coyuntura y la generación de actas notariales.

Palabras clave: Incendio; Anales; Diplomática; Buezo; Valpuesta; Campesinado; Donaciones.

1 Este trabajo se integra dentro de los proyectos de investigación El castellano norteño en la Edad Media (FF2016-80230-P) y Scriptoria, lenguajes y espacio agrario en la Alta Edad Media (HAR2017-86502-P), así como dentro del Grupo de Investigación Burgos-León-Valladolid. Fuentes escritas de Castilla y León (ss. VI-XVI) (BULEVAFUENTES) de la Universidad de Burgos. Quiero agradecer a los revisores anónimos sus acertados comentarios y sugerencias, así como a Iván García Izquierdo su ayuda en la redacción del texto. 
ABSTRACT: In the mid-tenth Century a wildfire ravaged the northern Meseta, and particularly Castile, leaving its mark not only in the narrative records of the moment and of successive generations, but also, rather exceptionally, in the diplomatic register where, in the months following the disaster, there would be a marked increase in activity. This is most keenly observed in an until now misunderstood dossier of twelve documents dating from the first half of 950 and referring to the monastery of Buezo which has been preserved in the Becerro Gótico of Valpuesta. This unusual coincidence between the annalistic and diplomatic genres, which has gone unnoticed until now, offers a unique insight into the nature of peasant life in times of crisis, and the relationship between such events and the generation of notarial acts.

Keywords: Wildfire; Annals; Diplomatics; Buezo; Valpuesta; Peasantry; Donations.

SUMARIO: 0 Introducción. 1 Un año nefasto. 2 Buezo. 3 El año 950 en otras colecciones diplomáticas. 4 Referencias bibliográficas. 5 Anexos.

\section{INTRODUCCIÓN}

A mediados del siglo x algo extraordinario ocurrió en la Meseta Norte que tuvo como foco especialmente intenso a la comarca burgalesa de la Bureba. Según la analística, se trataba de un incendio, de una intensidad y extensión inusitadas, a principios de verano. La conflagración también dejó su huella en la diplomática, análoga a una capa de ceniza en una estratigrafía arqueológica, aunque ha pasado desapercibida hasta ahora. Después, el recuerdo se fue modificando y confundiendo en diferentes géneros históricos, pseudo-históricos y legendarios. Esta inusual coincidencia entre la diplomática y la cronística abre un abanico de posibilidades analíticas: los ritmos anuales de la vida rural y los efectos que un desastre de tal calibre pudo tener en la familia campesina; la relación de los campesinos con las instituciones religiosas y la función caritativa de estas; y el proceso de creación de los diplomas y el volumen anual de los mismos.

\section{UN AÑO NEFASTO}

El extremo laconismo de los anales y su preocupación casi exclusiva por los grandes acontecimientos geopolíticos los convierten, normalmente, en una fuente de escasa utilidad para el estudio de la sociedad y economía campesinas. A modo de ilustración (Tabla 1), reproduzco a continuación el testimonio que ofrecen los Anales Castellanos Segundos para los casi tres siglos que siguieron a la invasión musulmana ${ }^{2}$. Como se puede apreciar,

2 Las fuentes empleadas para la elaboración de este trabajo son las siguientes (las presentamos precedidas de sus respectivas abreviaturas): Anales Castellanos, ed. Gómez-Moreno, Manuel. «Anales castellanos». En Discursos leídos ante la Real Academia de la Historia. Madrid: Real Academia de la Historia, 1917. Anales Compostelanos / Anales Castellanos Terceros, ed. Flórez, Henrique. España Sagrada: theatro geographico-historico de la iglesia de España, vol. XXIII (de la iglesia de Tuy). Madrid: Antonio Marín, 1767, p. 318. Becerro 
EL GRAN INCENDIO CASTELLANO DE 949. HUELLA DIPLOMÁTICA

Y MEMORIA HISTÓRICA DE UN DESASTRE NATURAL

DAVID PETERSON

la excesiva concisión, las ortografías irregulares, los acontecimientos casi exclusivamente geopolíticos y las cronologías inseguras son las principales características de este problemático género.

TABla 1. Extracto de los Anales Castellanos II, ed. Manuel Gómez-Moreno, 1917.

\begin{tabular}{|l|l|}
\hline 714 & venerunt sarraceni in Hispaniam tempore Ruderici regis, et preoccupaverunt eam set non totam \\
\hline 814 & exierunt forasmontani de Malakouria, et venerunt ad Castellam \\
\hline 788 & fregerunt cordubenses Soutuscovam \\
\hline 831 & populavit rex Ordonius Leionem \\
\hline 845 & populavit Rudericus comes Amaia \\
\hline 882 & populavit Didacus comes Burgus et Oiurna \\
\hline 912 & $\begin{array}{l}\text { populavit Munio Nunniz Roda et Gunzalvo Teliz Osma et Gunzalvo Fernandiz Cozça et Clunia et Sanctum } \\
\text { Stephanum secus fluvium Dorio }\end{array}$ \\
\hline 938 & venerunt sarraceni cum rege Adeffaman ad Setmancas \\
\hline 940 & populavit comde Fernan Gunzalviz Sedpublica \\
\hline 939 & sic fuit illo anno iniquo \\
\hline 983 & prendiderunt Setmancas \\
\hline 984 & prendiderunt Setpublica \\
\hline 986 & prendiderunt Zamora. Obiit famulus Dei Fernan Gunzalviz comes in mense iunii anno discurrente \\
\hline
\end{tabular}

Al margen de otros problemas cronológicos que se perciben, se aprecia cierta confusión en torno al periodo 938-940. Lo que nos interesa particularmente es esa referencia a un año iniquo, es decir, 'nefasto', fechado aquí en el año 939. Desde la perspectiva castellana, ¿en qué sentido podría considerarse nefasto el año 939, entendido en la mayoría de las fuentes como el de la gran victoria cristiana de Simancas? ¿A qué alude, entonces, esta breve y enigmática entrada?

En su reciente edición crítica del texto, J. C. Martín opta por introducir en la fecha una ' $L$ ' ausente del manuscrito y en un lugar muy poco habitual en el sistema de datación latina (Era DCCCCLXX[L]VII), para obtener la fecha de 959, argumentando que así se soluciona tanto el desorden cronológico de la secuencia como la incongruencia percibida

Galicano Digital (www.ehu.eus/galicano - consultado 03/08/2017). Cardeña = Colección documental del Monasterio de San Pedro de Cardeña, ed. Martínez Díez, Gonzalo. Burgos: Caja Círculo, 1998. Celanova = Colección diplomática del monasterio de Celanova (842-1230), eds. SÁEz, Emilio y SÁEz, Carlos. Alcalá de Henares: Universidad de Alcalá, 2000. Cronicón Burguense, ed. MartínEz Díez, Gonzalo. "Tres anales burgaleses medievales». Boletín de la Institución Fernán González, 2004, vol. 83, pp. 233-235. Cronicón de Cardeña, ed. Martínez Díez, Gonzalo. «Tres anales burgaleses medievales». Boletín de la Institución Fernán González, 2004, vol. 83, pp. 255-261. Gonzalo de Berceo. Vida de San Millán de la Cogolla. Edición y comentario, ed. Dutton, Brian. Madrid: Espasa-Calpe, 1992. León = Colección documental del Archivo de la Catedral de León (775-1230), ed. Sáez Sánchez, Emilio. León: Centro de Estudios e Investigación San Isidoro, 1987-1991. Poema de Fernán González, ed. Alarcos Llorach, Emilio. Madrid: Castalia, 1965. Sahagún = Colección diplomática del Monasterio de Sahagún, (siglos IX y X), ed. Mínguez Fernández, José María. León: Centro de Estudios e Investigación San Isidoro, 1976. Valpuesta = Los Becerros Gótico y Galicano de Valpuesta, 2 vols. eds. Ruiz Asencio, José Manuel; Ruiz Albi, Irene y Herrero Jiménez, Mauricio. Burgos: Instituto Castellano y Leonés de la Lengua, 2010. 
EL GRAN INCENDIO CASTELLANO DE 949. HUELLA DIPLOMÁTICA

142 Y MEMORIA HISTÓRICA DE UN DESASTRE NATURAL

entre la referencia a la iniquidad del año y la situación geopolítica del año $939^{3}$. El año 959, en cambio, sí se podría considerar nefasto desde la perspectiva castellana, argumenta Martín, por ser el del apresamiento del conde Fernán González por García Sánchez de Pamplona. Estamos de acuerdo en la necesidad de modificar la fecha -aunque sería conveniente confinar el ajuste propuesto a una nota a pie de página en vez de modificar la propia transcripción como hace Martín, eso sí, siempre señalando sus modificaciones-, pero sugerimos que la solución acertada es mucho más sencilla: introducir una ' $\mathrm{X}$ ' en vez de una ' $L$ '. El resultado es una secuencia mucho más natural dentro del sistema de datación latino-hispánico (Era DCCCCLXXXVII) que nos da la fecha de 949.

Para entender qué pudo ocurrir en 949 que justificara su calificación como iniquo, consultemos una segunda y paralela tradición analística, la de los Anales Castellanos Terceros $^{4}$. Con el laconismo característico del género, para las décadas centrales del siglo $\mathrm{x}$, estos terceros anales castellanos se limitan a relatar cuatro acontecimientos (Tabla 2). Llama la atención que estos apenas coincidan con los de los Anales Castellanos Segundos, así como la amplitud de la entrada del año 949.

TABLA 2. Comparación de diferentes tradiciones analísticas castellanas.

\begin{tabular}{|c|c|}
\hline Anales Castellanos Segundos & Anales Castellanos Terceros (Compostelanos) \\
\hline & $\begin{array}{l}925 \text { martyrizatus est B. Pelagius in Cordoba, et in Era } \\
\text { MV translatum est corpus eius de Cordoba per Blasium } \\
\text { episcopum, et reconditum est honorifice apud Legionem }\end{array}$ \\
\hline \multicolumn{2}{|l|}{$\begin{array}{l}938 \text { venerunt sarraceni cum rege Adeffaman ad } \\
\text { Setmancas }\end{array}$} \\
\hline \multicolumn{2}{|l|}{940 populavit comde Fernan Gunzalviz Sedpublica } \\
\hline \multicolumn{2}{|l|}{939 sic fuit illo anno iniquo } \\
\hline & $\begin{array}{l}949 \text { flamma exivit de mari et incendit plurimas urbes, et } \\
\text { villas, et homines, et bestias, et in ipso mari pinnas incendit: } \\
\text { et in Zamora unum barrium, et in Carrion, et in Castroxeriz, } \\
\text { et in Burgos C. casas, et in Birbiesca, et in Calzada, et in } \\
\text { Pontecorvo, et in Buradon et alias plurimas villas combusit }\end{array}$ \\
\hline & $\begin{array}{l}960 \text { fuit captus comes F. Gonzalvi et filii eius in Aconia à rege } \\
\text { Garsia, et transmisit illos in Pampillis }\end{array}$ \\
\hline & 970 obiit Ferdinandus Gundisalvi \\
\hline 983 prendiderunt Setmancas & \\
\hline
\end{tabular}

Existen otras versiones del relato que introducen algunas variantes. Por ejemplo, el Cronicón Burguense que, a pesar de su nombre, no es una crónica, ni mucho menos una gran crónica, sino otra muestra más del género anales ${ }^{5}$. Como se puede apreciar, la

3 Martín Iglesias, José Carlos. «Los Annales Castellani Antiquiores y Annales Castellani Recentiores: edición y traducción anotada». Territorio, Sociedad y Poder, 2009, vol. 4, pp. 203-226, aquí p. 219.

4 Aunque por su contenido se aprecia un origen castellano, estos anales también son conocidos como Compostelanos debido a su conservación dentro del Tumbo Negro de Santiago de Compostela; FLórez, Henrique. España Sagrada: theatro geographico-historico de la iglesia de España, vol. XXIII (de la iglesia de Tuy). Madrid: Antonio Marín, 1767, p. 318.

5 De hecho, Martínez Díez proponía designarlos como los Annales Burgenses. Martínez DíEz, Gonzalo. «Tres anales burgaleses medievales». Boletín del Instituto Fernán González, 2004, vol. 83, pp. 227-263, especialmente p. 230. 
EL GRAN INCENDIO CASTELLANO DE 949. HUELLA DIPLOMÁTICA

Y MEMORIA HISTÓRICA DE UN DESASTRE NATURAL

DAVID PETERSON

redacción es prácticamente idéntica a la de los Anales Castellanos Terceros con algunas mínimas, aunque significativas, diferencias: el año es ahora 939; se añade el día de la semana, el del mes e incluso la hora del incendio; se omite la referencia a las 100 casas de Burgos; y se prescinde del verbo final combusit.

Era DCCCCLXXVII kalendas iunii die sabbati hora IX a flamina exiuit de mari, et incendit plurimas villas et urbes, et homines, et bestias, et in ipso mari pinnas incindit et in Zamora unum barrium et casas plurimas et in Carrión et in Castro Xorit et in Burgis et in Beruiesca et in Calçda et in Panticorvo, et in Buradon et alias plurimas villas ${ }^{6}$.

También disponemos de una versión romanceada conservada en el llamado Cronicón de Cardeña, o más acertadamente Anales de Cardeña como proponía G. Martínez ${ }^{7}$ y que combina elementos de las dos versiones latinas:

Era de DCCCCLXXXVII, kalendas juni dia de sábato a hora de nonna, salió flama del mar et ençendió muchas villas et cibdades et omnes et bestias; et este mismo mar ençendió pennas et en Çamora I barrio et en Carrión, en Castro Xeriz et en Burgos C casas et en Briuesca et en Calçada et en Pancoruo, et en Buradón et otras muchas villas ${ }^{8}$.

Al margen de las mínimas variaciones recogidas en la Tabla 3, las tres versiones coinciden en lo esencial del relato: un incendio que sale del mar antes de extenderse desde el oeste e incidir de manera muy notable en la provincia de Burgos y, con especial virulencia, en la comarca de la Bureba. Cabe señalar aquí que Calzada no se refiere al burgo riojano de Santo Domingo (que todavía no se había fundado en el siglo x) sino a un homónimo pueblo burebano, situado entre Briviesca y Pancorbo, como el mismo relato indica. Que se incluya esta intranscendente aldea en la lista sugiere una inusitada concentración del fenómeno en la Bureba.

TABLA 3. Diferencias entre las tres versiones analísticas del incendio.

\begin{tabular}{|c|l|l|l|}
\hline & $\begin{array}{c}\text { Anales Castellanos III } \\
\text { (A. Compostelanos) }\end{array}$ & \multicolumn{1}{c|}{$\begin{array}{c}\text { Anales Burguenses } \\
\text { (Cronicón de Burgos) }\end{array}$} & \multicolumn{1}{c|}{$\begin{array}{c}\text { Anales Cardenienses } \\
\text { (Cronicón de Cardeña) }\end{array}$} \\
\hline Año & DCCCCLXXXVII [949] & DCCCCLXXVII [939] & DCCCCLXXXVII [949] \\
\hline Día & - & kalendas iunii die sabbati hora IXa & $\begin{array}{l}\text { kalendas juni dia de sábato a hora } \\
\text { de nonna }\end{array}$ \\
\hline Burgos & et in Burgos C. casas & et in Burgis et in ... & et en Burgos C casas \\
\hline Verbo final & combusit & - & - \\
\hline
\end{tabular}

Fuera de la tradición analística, la memoria del desastre se fue incorporando en otros géneros como la diplomática y la poesía épica. En ambos casos se pretendió dotar

6 Cronicón Burguense, Archivo de la Catedral de Burgos, vol. 73, fols. 162-163. Publicado en MArTíNEZ DíEZ, «Tres anales burgaleses medievales», p. 234.

7 Martínez DíEz, «Tres anales burgaleses medievales», p. 255.

8 Martínez Díez, «Tres anales burgaleses medievales», p. 256. También en Flórez, España Sagrada. vol. XXIII, p. 370. 
al hecho de un significado histórico más amplio, relacionándolo con las guerras entre castellanos y andalusíes que caracterizaron gran parte del siglo x. Así, en los llamados Votos de San Millán, un diploma apócrifo redactado hacia finales del siglo XII, se asoció el incendio con una serie de fenómenos naturales que precedieron la batalla de Simancas, sobre todo un eclipse solar (lumen solis ... obscuratum) constatado históricamente en otras fuentes y acaecido el 19 de julio de $939^{\circ}$, aunque fechado en el falso emilianense en el año 934. Aquí el incendio se describe como una puerta en llamas que se abrió en el cielo (porta flamea aperta est in celo), empujado por un viento sur (vento africo) y que quemó gran parte de la tierra (magnam terre partem conbussit) ${ }^{10}$.

Los términos genéricos y portentosos con que se describe el incendio en los Votos, añadido a la migración de la fecha a 934, podrían hacernos dudar que se tratara del mismo fenómeno que la conflagración relatada por la analística. Sin embargo, tales dudas desaparecen cuando se lee la versión de los mismos hechos en la Vida de San Millán (c 1225), obra de Gonzalo de Berceo, un monje del monasterio homónimo. Berceo también relaciona esta gran conflagración con los portentos celestiales anteriores a la batalla de Simancas, pero su versión versificada es mucho más extensa que la de los Votos e incorpora una descripción geográfica del acontecimiento claramente relacionada con la tradición analística, aunque incluso más amplia que esta. Es evidente que se trata del mismo acontecimiento. Lo que no está claro es si las adiciones de Berceo provienen de otra fuente desconocida o de su propia imaginación, pero, al añadirse una nueva referencia a diez casas quemadas en Pancorbo, se refuerza la idea de la concentración del incendio en lo que hoy es la provincia de Burgos:

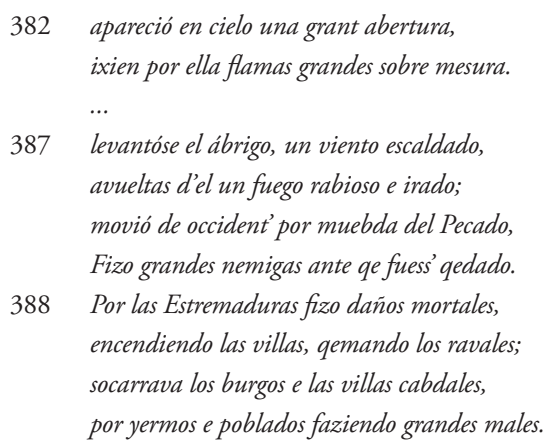

9 El eclipse se menciona también en los Anales Castellanos Primeros, en la Crónica de Sampiro e incluso en fuentes carolingias como los Annales Sangallenses; Martín Iglesias, «Los Annales Castellani», p. 211, n. 60 .

10 In terra apparuerunt signa quod furor Domini venturus credebatur esse in ea. In era noningentesima septuagesima secunda, XIIII" kalendas augusti, lumen solis die VIa feria, amittens lucendi virtutem, obscuratum constitit ab hora secunda in tertiam; IIII feria idus octobris, colorem eiusdem solis multi cognoverunt effectum pallidum. Signa magna facta est in celo vento africo. Porta flamea aperta est in celo, et ibant stelle et commovebant se huc adque illuc, maxime plus discurrebant contra vento africo, et mirate sunt gentes de his signis noctis media usque mane. Et fumificus vapor magnam terre partem conbussit (Becerro Galicano Digital \#0, http://www.ehu. eus/galicano/id0\&l=es\&cro $=0$ ) 
EL GRAN INCENDIO CASTELLANO DE 949. HUELLA DIPLOMÁTICA

Y MEMORIA HISTÓRICA DE UN DESASTRE NATURAL

DAVID PETERSON

389 Plegó a Santfagunt qemó una partida,

fue cerca de la media de Carrión ardida;

por poco fuera toda Frómesta consumida,

Castro entre las otras non remanso senzida.

390 Forniellos del Camino fincó mal socarrada,

Oterdajos qe yaze en una renconada;

acorrióli a Burgos qe yazié derramada,

ca non era estonz' en un logar poblada.

391 Qemó en Monesterio quanto delante priso,

en Pancorbo diez casas, ca parcir no li quiso,

muchos otros logares qe en carta non miso

en qui fizo el fuego escarnio e grand riso.

Gonzalo de Berceo, Vida de San Millán, ed. B. Dutton, 1992.

Por último, en el Poema de Fernán González, elaborado a mediados del siglo XIII, siguiendo la tradición emilianense el incendio se identifica plenamente con la decisiva victoria de Fernán González sobre los musulmanes, aunque anacrónicamente estos ahora incorporan en su hueste a turcos (\#389), su caudillo es Almanzor (\#387), y la batalla se desarrolla en Hacinas (\#395). Poéticamente, la conflagración se ha convertido ya en obra de una inmensa serpiente ignívoma, producto de la diabólica magia morisca (\#482):

471 Vieron aquella noche una muy fiera cosa:

venia por el aire una sierpe rabiosa,

dando muy fuertes gritos la fantasma astrosa

oda venía sangrienta, bermeja como rosa.

472 Ella tenía el aspecto de que herida venía,

parecía que el cielo, con sus gritos, partía;

alumbraba las huestes el fuego que vertía:

todos tuvieron miedo que a quemarlos venía.

473 No hubo nadie entre ellos de alma tan esforzado

que no tuviera miedo y no fuese espantado;

cayeron muchos hombres en tierra del espanto,

tuvo muy gran temor todo el pueblo cruzado.

Anónimo, Poema de Fernán González, ed. E. Alarcos, 1965.

Así, la llama que salió del mar se ha convertido en una sierpe rabiosa -le queda todavía otra transformación sorprendente-, pero las fuentes, tanto latinas como romances, analísticas y poéticas, están de acuerdo en que se trataba de un incendio devastador, con una incidencia especial en Castilla y particularmente en la Bureba. Con las sucesivas iteraciones del relato, lo que en la analística había sido una noticia exenta, llamativamente desarrollada y geográficamente muy específica, se ha ido confundiendo con los acontecimientos que rodearon la batalla de Simancas, primero como "portento divino» (Votos, Vida), después como «magia negra morisca» (Poema), consolidándose así la fecha de 939, o incluso la de 934 en la tradición emilianense (Tabla 4). 
EL GRAN INCENDIO CASTELLANO DE 949. HUELLA DIPLOMÁTICA Y MEMORIA HISTÓRICA DE UN DESASTRE NATURAL DAVID PETERSON

TABLA 4. La progresiva mitificación del incendio.

\begin{tabular}{|l|c|l|}
\hline \multicolumn{1}{|c|}{ Fuente } & FeCha ATRIBUida & \multicolumn{1}{c|}{ ConTeXTo } \\
\hline Annales Compostellani & 949 & Hecho histórico aislado \\
\hline Cronicón Burguense & 939 & Hecho histórico aislado \\
\hline Cronicón de Cardeña & 949 & Hecho histórico aislado \\
\hline Votos de San Millán & 934 & Batalla de Simancas \\
\hline Vida de San Millán & 934 & Batalla de Simancas \\
\hline Poema de Fernán González & - & Batalla de Hacinas \\
\hline Pérez de Urbel (1970) & 939 & Batalla de Simancas \\
\hline Martínez Díez (2006) & {$[939]$} & «rechazamos la historicidad de tal hecho» \\
\hline
\end{tabular}

El proceso de migración cronológica se culmina en la historiografía castellana del siglo xx, cuando Pérez de Urbel asociaba el incendio plena e históricamente con la batalla de Simancas, silenciando cualquier referencia a $949^{11}$. El último paso lo dio Martínez Díez, que también manejaba la fecha de 939, pero rechazó dogmáticamente la historicidad del incendio por el argumento circular de que su ausencia de los Primeros Anales Castellanos le restaba verosimilitud histórica: pues, siempre según Martínez, redactados estos en 940, «al año siguiente del pretendido prodigio», cómo no iban a mencionar tan prodigioso hecho ${ }^{12}$. Aunque anteriormente había mencionado que son tres los cronicones que recogen el suceso, solo cita la fecha de 939 recogida por el Burguense, callando, al igual que había hecho Pérez de Urbel en su día, las referencias a 949, una fecha que, sin embargo, habría solventado el problema percibido de su ausencia de los Primeros Anales Castellanos. Al margen de esta cuestionable metodología, observamos cómo el incendio se ha ido fundiendo y confundiendo con el eclipse de 939 y se ha mitificado un fenómeno histórico que va camino de la marginación historiográfica completa.

No obstante, la memoria del incendio sobrevive en un contexto inesperado: según el Ministerio de Fomento fue provocado por un terremoto ${ }^{13}$. La idea parece haber arraigado primero en la obra de una serie de eruditos zamoranos decimonónicos ${ }^{14}$, uno de los cuales incluso asoció el incendio con la erupción de un volcán submarino ${ }^{15}$, para penetrar

11 Pérez de Urbel, Justo. El condado de Castilla. Madrid: Siglo Ilustrado, 1969-1970, 3 vols.; aquí vol. II, p. 111.

12 Martínez Díez, Gonzalo. El condado de Castilla. (711-1038). La Historia frente a la Leyenda. Valladolid-Madrid: Junta de Castilla y León-Marcial Pons, 2005, aquí vol. 2, pp. 342-343.

13 Martínez Solares, José Manuel y Mezcua, Julio. Catálogo sísmico de la Península Ibérica (880 a. C.-1900). Madrid: Dirección General del Instituto Geográfico Nacional, 2002.

14 Fernández Duro, Cesáreo. Memorias históricas de la ciudad de Zamora. Madrid: Sucesores de Rivadeneyra, 1882; Puig y Larraz, Gabriel. Descripción física y geológica de la provincia de Zamora. Madrid: Manuel Tello, 1883, pp. 164-168; Calvo Madroño, Ismael. Descripción geográfica, histórica y estadística de la provincia de Zamora. Madrid: Imprenta de Victoriano Suárez, 1914.

15 «La tierra fue teatro por esta parte de violentísima conmoción atribuida por unos á la erupción de un volcán submarino que arrojando impetuosamente las aguas mezcladas de lavas hirvientes y en ignición sobre las costas y por las desembocaduras de los ríos contrariando las corrientes», ÁLvarez MartíneZ, Ursicino. Historia general de la provincia de Zamora. Zamora: La Seña Bermeja, 1889, aquí p. 128. 
EL GRAN INCENDIO CASTELLANO DE 949. HUELLA DIPLOMÁTICA

Y MEMORIA HISTÓRICA DE UN DESASTRE NATURAL

DAVID PETERSON

después en los catálogos oficiales de actividad sísmica peninsular ${ }^{16}$. De allí pasaría a la divulgación en lugares como Wikipedia ${ }^{17}$. Todo esto sin que se aporte más indicio de tal terremoto que los relatos analísticos que, como hemos visto, no mencionan en ningún momento tal fenómeno. Aunque es cierto que históricamente los terremotos han sido la causa de devastadores incendios, generalmente esto ha ocurrido en contextos urbanos, siendo el ejemplo más notorio el desastre de Lisboa de 1755. Pero, además de la completa falta de testimonios de tal terremoto, la principal objeción contra la hipótesis de un origen sísmico es la realidad geológica de la Meseta Norte, prácticamente inerte en estos términos ${ }^{18}$, tal y como los citados registros de sismicidad histórica del Ministerio de Fomento demuestran año tras año, con 949 como prácticamente la única excepción. Así que, como causa del incendio, un terremoto es casi tan inverosímil como el dragón del Poema de Fernán González.

Llama la atención la disposición lineal de los lugares afectados por la conflagración, los cuales siguen las principales vías romanas de la Meseta Norte. Dado que estas fueron aprovechadas tradicionalmente por las aceifas musulmanas, esta alineación podría sugerir un origen bélico del incendio. No obstante, en otras ocasiones los anales describen tales campañas sin necesidad de recurrir a la metáfora, y tampoco hay eco de una expedición de estas características (año, dirección, grado de devastación) en la cronística árabe, por ejemplo, en la de Ibn-Idhari, por citar un autor que aporta testimonios relativos a esas fechas.

Si buscamos explicaciones para la conflagración fuera de la Península, el paralelo histórico más parecido a lo relatado en los Anales Castellanos sería los macro-incendios del 8 de octubre de 1871 ocurridos simultáneamente en Chicago (Illinois), Port Huron (Michigan) y Peshtigo (Wisconsin), separados entre sí por $400 \mathrm{~km}$ de distancia. Para estos incendios norteamericanos se postula un origen en la coyuntura de la acumulación de material inflamable como consecuencia de un proceso de deforestación rápida y descontrolada, temperaturas extremas y vientos fuertes, cuyo resultado sería una combustión politópica pero prácticamente sincrónica ${ }^{19}$. No han faltado propuestas explicativas

16 Galbis Rodríguez, José. Catálogo sísmico de la zona comprendida entre los meridianos $5^{\circ}$ E y $20^{\circ}$ Wy paralelos $45^{\circ}$ y $25^{\circ} \mathrm{N}$. Madrid: Instituto Geográfico, Catastral y de Estadística, 1932, p. 807; Munuera QuiÑonero, José María. A study of seismicity on the Peninsula Ibérica area. Technical note n. 1 "Seismic Data". Madrid: Instituto Geográfico y Catastral, 1963; Mezcua, Julio y Martínez Solares, José Manuel. Sismicidad del área iberomogrebi. Madrid: Instituto Geográfico Nacional, 1983, p. 301.

17 «Dado que en la lacónica información dada en los antiguos cronicones no se le reconoce explícitamente como un terremoto, algunos cronistas del s. xvi conjeturaron que el episodio pudo ser una invención popular hecha sobre algunos incendios accidentales, o que pudo estar causado por «algin aspecto maligno de las estrellas». Pero historiadores y geólogos posteriores lo interpretaron como un movimiento sísmico, y como tal se le considera actualmente» [Wikipedia, consultado, 21-06-2017].

18 Martinez Solares, José Manuel. «Sismicidad histórica de la Península Ibérica». Física de la Tierra, 2003, vol. 15, pp. 13-28. Muy amablemente este autor me ha facilitado el acceso a un informe inédito sobre la cuestión (Estudio de determinación de los datos sismicos de base para las obras hidráulicas, firmado por «Ingeniería 75, S. A.») encargado en 1986 por el Ministerio de Obras Públicas y Urbanismo y que cuestiona seriamente la historicidad del terremoto (que asocia con el 939), «en consecuencia opinamos que resulta bastante aventurado suponer la existencia de un terremoto en julio o agosto de 939» (p. 49).

19 Pyne, Stephen J. Fire in America. A Cultural History of Wildland and Rural Fire: Princeton: Princeton University Press, 1982. 
EL GRAN INCENDIO CASTELLANO DE 949. HUELLA DIPLOMÁTICA Y MEMORIA HISTÓRICA DE UN DESASTRE NATURAL

alternativas para los incendios norteamericanos. La idea de un origen celestial (meteorito, cometa) arraigó con fuerza en la imaginación popular y, aunque generalmente rechazada como causa por la literatura científica (en el caso americano), explicaría la distribución lineal de los núcleos meseteños afectados.

Al margen de la causa del incendio, queda por dirimir la cuestión de su cronología. En primer lugar, creo que se debe entender a las versiones analísticas, libres de pretensiones literarias, como nuestras fuentes más antiguas para este acontecimiento. Se ha venido datando estas obras a partir de la fecha de su última entrada y con referencia también al tipo de letra empleada, lo cual daría al Cronicón Burguense, por ejemplo, una fecha de composición hacia el segundo cuarto del siglo $\mathrm{XIII}^{20}$. Ello lo haría posterior al texto de los Votos y coetáneo de la obra de Berceo. No obstante, parece más razonable pensar en términos de una composición diacrónica para los anales, con entradas más o menos contemporáneas a los hechos narrados, como el propio nombre del género indica, y como se comprende para los Anales de San Bertín o los de Fulda ${ }^{21}$. Efectivamente, para el caso que nos ocupa, la irregularidad de estilo y el tamaño de las entradas indican claramente que no es una obra unitaria (realizada íntegramente en el siglo XIII), sino el resultado de un proceso de composición diacrónica. Más específicamente, el lenguaje empleado en la entrada que nos interesa, tanto en el Cronicón Burguense como en los Anales Castellanos $I I I$, es característico del siglo $\mathrm{x}$, con formas verbales latinas, pero sintaxis y pluralización de sustantivos claramente romances. Esta forma de plasmar en escritura el primitivo romance es entendida por Wright "cómo se escribía el romance antes de que el romance se escribiera» ${ }^{22}$, y es completamente ajeno al siglo XIII cuando ya se hace una distinción consciente entre latín y castellano, como el contraste entre las dos versiones de los Votos demuestra.

Admitidos los anales como las fuentes más antiguas, podemos desechar la fecha de 934 ofrecida en los textos emilianenses y nos queda elegir entre 939 y 949 como año más verosímil para el incendio. El hecho de que el 1 de junio cayera en viernes en 949 y en sábado en 939, nos inclina inicialmente hacia la data más temprana, dada la aportación al respecto del Cronicón Burguense. No obstante, 949 parece más convincente por otra serie de razones que enumeraré a continuación, y sospecho que el día de la semana fuera una introducción tardía ausente de una hipotética versión primigenia de los hechos.

El primer motivo para favorecer la fecha de 949 es su intranscendencia histórica. Solo en los géneros más literarios se asocia el incendio con la batalla de Simancas. Entiendo esto como una reinterpretación tardía, mediante la conflación de dos tradiciones, en un intento de dotar el incomprendido desastre de un sentido histórico, migrando así la fecha hacia acontecimientos bélicos notorios y condicionado por el hecho de que la contienda de Simancas ya se asociaba con portentos cósmicos históricamente contrastados.

20 Martínez Díez, «Tres anales burgaleses medievales», p. 231.

21 Nelson, Jinty. The Annals of St-Bertin. Manchester: Manchester University Press, 1991, pp. 4-5; Reuter, Timothy. The Annals of Fulda. Manchester: Manchester University Press, 1992, pp. 4-5.

22 Wright, Roger. "Cómo se escribía el romance antes de que el romance se escribiera», conferencia inédita pronunciada como parte del coloquio Recuperar el cartulario, celebrado en San Millán de la Cogolla, 9 de octubre de 2013. Una versión inglesa está disponble como «How Scribes Wrote Ibero Romance Before Ibero Romance was Invented", https://liverpool.academia.edu/RogerWright. 
EL GRAN INCENDIO CASTELLANO DE 949. HUELLA DIPLOMÁTICA

Y MEMORIA HISTÓRICA DE UN DESASTRE NATURAL

DAVID PETERSON

En cambio, no se aprecia motivo alguno para trasladar un hecho que hipotéticamente tuviera lugar en el 939 al históricamente insignificante año 949.

También apoya una fecha original en la década de los 940 la comentada aparición en los Anales Castellanos Segundos de la enigmática entrada anno iniquo fuera de secuencia cronológica. Además, ese acontecimiento iniquo se muestra como un hecho expresamente diferenciado de la batalla de Simancas (aquí fechada en 938), una victoria que difícilmente valdría para tildar un ańo como nefasto desde la perspectiva castellana. Todos estos problemas se resuelven si el año iniquo fuese en origen el 949, fácilmente cambiado a 939 en un error de transmisión. Esta brevísima aportación de los Anales Castellanos Segundos es especialmente valiosa porque supone el recuerdo de un acontecimiento nefasto posterior a Simancas y muy posiblemente en la década de los 940 en lo que sería una segunda e independiente tradición cronística al margen de la de las otras tres versiones, las cuales parecen tener todas ellas un origen común. A continuación veremos que un acontecimiento singularmente traumático que coincide, grosso modo, con la geografía del incendio parece tener eco también en la diplomática del periodo y así confirma definitivamente la fecha de 949.

\section{Buezo}

Este epígrafe remite, sobre todo, a una secuencia excepcional de documentos que se ha preservado en los folios 82r-92r del Becerro Gótico de Valpuesta ${ }^{23}$. En una fuente en la que durante las décadas centrales del siglo $\mathrm{x}$ lo habitual es que se conserve un documento cada dos años, repentinamente apreciamos que para el año 950 hay una docena (Gráfico 1). Además, se da la circunstancia de que todos ellos refieren al mismo pueblo burebano de Buezo, próximo a Briviesca. Contextualicemos esto en términos cuantitativos: para toda Castilla han sobrevivido apenas 200 documentos para el siglo $\mathrm{x}$ y, de repente, doce de ellos corresponden a un solo año en un mismo pueblo burebano ${ }^{24}$. En toda Castilla no hay otra colección tan nutrida ni tan temprana para una sola localidad y, menos aún, para un solo año.

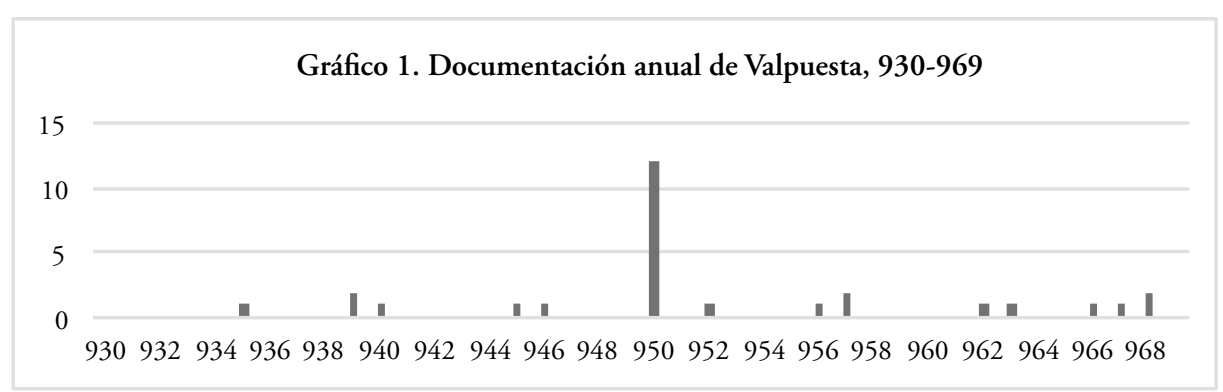
20-31.

23 Ruiz Asencio, Ruiz Albi y Herrero Jiménez, Los Becerros Gótico y Galicano de Valpuesta, docs.

24 Este cartulario de Buezo de Bureba (Ruiz Asencio et álii, 47) es también insólito en el sentido de que los fondos valpositanos raras veces proporcionan información sobre espacios tan meridionales; y Buezo, en concreto, no volverá a figurar en toda la documentación valpositana. 
La bibliografía sobre este singular dosier es escueta. Peña Pérez se fijó en el patético testimonio de extrema hambruna contenido en uno de los textos como ejemplo de la precariedad vital del campesinado en este periodo, pero no analizó la serie en su conjunto ${ }^{25}$. Davies se limitó a comentar que tanta concentración de donaciones campesinas es atípica ${ }^{26}$, mientras en su análisis de la antroponimia de la serie Ramos remarcó su naturaleza vascónica occidental ${ }^{27}$. Para Zabalza, estos doce textos suponían los «restos de un cartulario desaparecido", y este autor recomendó su estudio ${ }^{28}$. Ruiz Asencio describió estos documentos como "compras necesarias que hace su abad Gonzalo para constituir el patrimonio inicial de la institución ${ }^{29}$, un extraño análisis dado que son mayoritariamente donaciones. En 2010 y firmando ya con su equipo de edición de la documentación valpositana, este mismo autor dedicó varios pequeños apartados al conjunto en sus páginas: 54-55 (estudio codicológico), 86-87 (estudio paleográfico) y 135-137 (estudio diplomático). Rectificando, y alejándose de la afirmación realizada en 2004 de que se tratara del patrimonio fundacional del monasterio burebano, sugirió que el dosier podría representar el «movimiento de documentos que se producía en algo más de medio año en el archivo monástico ${ }^{30}$. Desconocemos la existencia de otras aproximaciones al dosier.

Esta lectura resulta muy problemática por sus implicaciones en cuanto al volumen de transacciones registradas por un monasterio tan modesto como Buezo que, al margen de este dosier, no ha dejado apenas huella. ¿Debemos entender que todos los monasterios generaban tal volumen de documentación en un año cualquiera? Parece improbable, pero tampoco explicaría por qué sobrevivió esta colección y no los otros miles de hipotéticos documentos que esta teoría presupone. Parece más verosímil que este volumen de documentación se deba a un proceso de creación extraordinario causado, a su vez, por un hecho también extraordinario. Y esto parece confirmarse cuando se observan dinámicas parecidas en otras colecciones documentales.

\section{El AÑo 950 EN OTRAS COLECCIONES DIPLOMÁTICAS}

En el siguiente análisis de diferentes colecciones diplomáticas de la Meseta Norte solo se ha tenido en cuenta transacciones particulares, excluyéndose la actividad regia o episcopal, los litigios y las permutas institucionales. Las grandes colecciones diplomáticas

25 Peña Pérez, Francisco Javier. «Los espacios sociales en la España cotidiana. Valpuesta en la Edad Media». En MCC Aniversario del obispado de Valpuesta: 804-2004. Miranda de Ebro: Fundación Cultural Miranda de Ebro, 2004, pp. 291-319, aquí p. 306-307.

26 Davies, Wendy. Acts of Giving. Individual, Community, and Church in Tenth-Century Christian Spain. Oxford: Oxford University Press, 2007, aquí p. 210.

27 Ramos Remedios, Emiliana. «Francos y vascos en el norte de Castilla (IX-XIII): los cambios en las denominaciones personales». Nouvelle Revue d'Onomastique, 2014, vol. 56, pp. 197-225, aquí p. 215.

28 Zabalza Conde, Manuel. «Tipología de los documentos de Valpuesta». En MCC Aniversario del Obispado de Valpuesta, pp. 320-353, aquí p. 321.

29 Ruiz Asencio, José Manuel. «Los cartularios de Valpuesta». En MCC Aniversario del Obispado de Valpuesta, pp. 354-381, aquí p. 363.

30 Ruiz Asencio, Ruiz Albi y Herrero Jiménez, Los Becerros Gótico y Galicano de Valpuesta. La cita es de la página 87. 
EL GRAN INCENDIO CASTELLANO DE 949. HUELLA DIPLOMÁTICA Y MEMORIA HISTÓRICA DE UN DESASTRE NATURAL DAVID PETERSON

de la Meseta Norte consisten mayoritariamente de donaciones regias y aristocráticas hasta aproximadamente 930 que es cuando comienza a aparecer una proporción significativa de transacciones campesinas ${ }^{31}$. Consecuentemente, para contextualizar lo documentado en 950, tomaremos como referencia el periodo comprendido entre 930-969.

La segunda mayor concentración que encontraremos entre toda la diplomática castellana del siglo x se produce en el Becerro Gótico de San Pedro de Cardeña y de nuevo corresponde al ańo 950, con 14 transacciones ${ }^{32}$. La concentración cardeniense no es tan llamativa (y por tanto no ha llamado tanto la atención) ya que aparece en un fondo mucho más amplio -con una media de tres documentos por año en este periodo- que la de Valpuesta (Gráfico 2). A diferencia del dosier de Buezo, este pico de actividad tampoco se limita a una sola aldea, pues Cardeńa recibe donaciones en varias aldeas próximas, además de recoger noticia de otras tres dádivas dirigidas a San Andrés de Villalbilla, a unos siete kilómetros al suroeste de Burgos, un segundo pequeńo monasterio que surge repentinamente del silencio documental, a semejanza de Buezo.

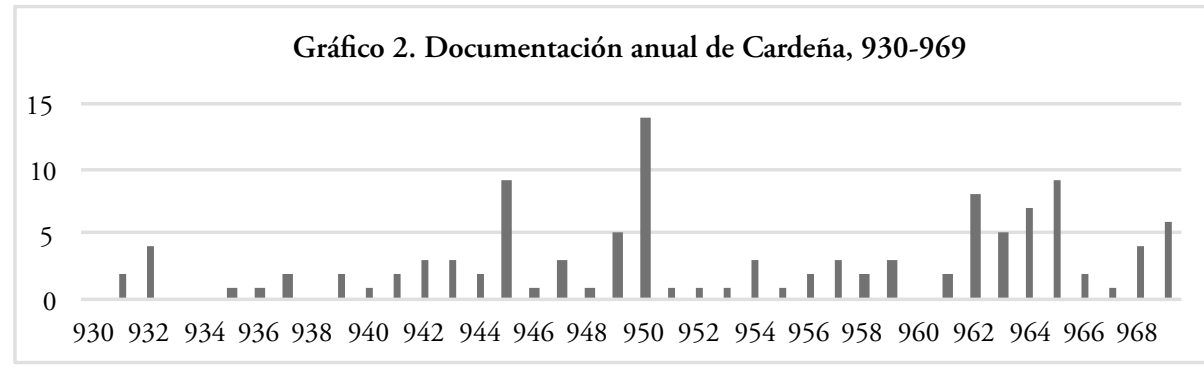

La combinación de este pico de actividad diplomática en Cardeña con la de Buezo es lo que llama la atención. Si la supervivencia del dosier de Buezo ha sido considerada fruto de un accidente archivístico, la existencia de una concentración parecida en otro archivo debilita enormemente esa lectura. Juntos reflejan necesariamente algo más que un accidente -en este caso de supervivencia- dentro de un solo archivo (Gráfico 3).

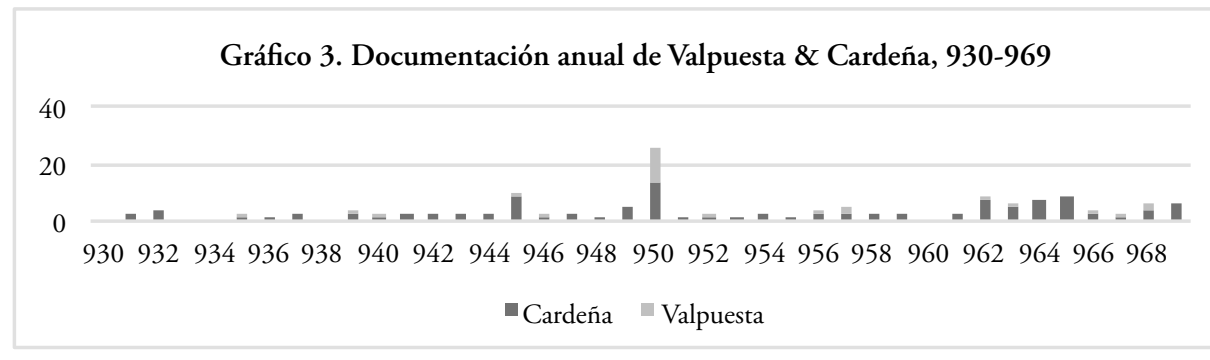

31 Davies, Acts of Giving, p. 210.

32 Martínez Díez, Gonzalo. Colección documental del Monasterio de San Pedro de Cardeña. Burgos: Caja Círculo, 1998, docs. 67-80. 
Es en las fuentes burgalesas donde más se nota la anomalía del 950, pero más al oeste -en la documentación de Sahagún y León- también se aprecia que el 950 fue un año inusitadamente propicio para la generación (o, menos plausiblemente, conservación) de documentación. Aunque en estas tierras ya leonesas el contraste con otros ańos es algo menos dramático, con la excepción de otro pico de actividad en la documentación de Sahagún en $965^{33}$, 950 sigue siendo el año de mayor actividad diplomática (Gráfico 4) ${ }^{34}$. Por lo tanto, podemos concluir que la superproducción diplomática es un fenómeno generalizado en la Meseta Norte ese año, lo cual lo aleja de cualquier explicación referente a la supervivencia o transmisión documental del tipo sugerido por Ruiz Asencio. Es más, en realidad, aunque reunidas en cuatro grandes colecciones diplomáticas, estamos ante una actividad aún más fragmentada y diversificada, con documentos provenientes de Villalbilla incluidos en el fondo de Cardeńa, y textos de Abellar y Melgar conservados en la documentación leonesa.

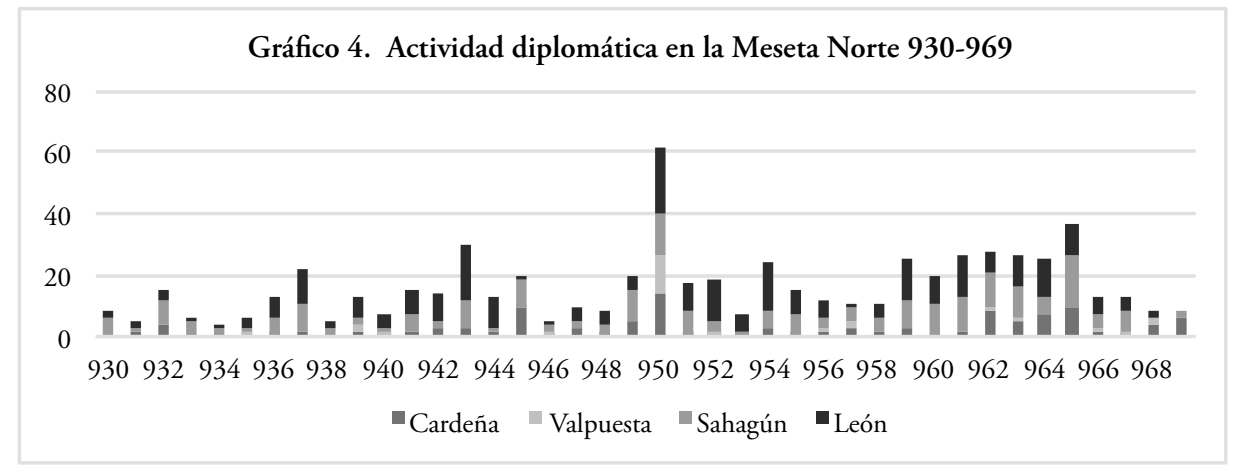

Mientras Sahagún sí figura en la geografía del incendio ofrecida por la analística, no ocurre lo mismo con León. No obstante, lo incluimos aquí como elemento de control para comprobar si el fenómeno de superproducción diplomática se limita a la geografía indicada por la analística y, en general, para aumentar nuestro muestreo y consolidar así nuestra comprensión de los ritmos anuales de producción diplomática. De todos modos, aunque alejado de la geografía del incendio relatado en la analística, entendemos que las previsiblemente extremas condiciones climatológicas que favorecerían el desarrollo del

33 La actividad de 965 (Sahagún224 a 241) se concentra como la de 950 en el primer semestre del año, pero se limita casi exclusivamente (17 de 18 textos, todos ellos son del primer semestre) a una sola aldea, Valdefresno. Tal concentración es en sí muy llamativa, pero habría que contemplar una causa más localista que la de 950 .

34 Mínguez Fernández, José María. Colección diplomática del Monasterio de Sahagún (siglos IX y X). León: Centro de Estudios e Investigación San Isidoro, 1976, docs. 94 (este contiene varias transacciones de diferentes años), 120-122, 124-125 y 127; SÁEz SÁnchez, Emilio. Colección documental del Archivo de la Catedral de León (775-1230). León: Centro de Estudios e Investigación San Isidoro, 1987-1991, docs. 210219 y 221-230. Más al oeste, en cambio, 950 no parece haber sido un año excepcional según SÁEz, Emilio y SÁEz, Carlos. Colección diplomática del monasterio de Celanova (842-1230). Alcalá de Henares: Universidad de Alcalá, 2000. 
EL GRAN INCENDIO CASTELLANO DE 949. HUELLA DIPLOMÁTICA Y MEMORIA HISTÓRICA DE UN DESASTRE NATURAL DAVID PETERSON

incendio podrían tener también consecuencias negativas en comarcas cercanas no directamente implicadas en la conflagración.

Si ya de por sí es llamativa la concentración de actividad en 950, lo es más cuando observamos cómo la concentración se da de una manera aún más pronunciada en los primeros seis meses del año (Gráfico 5). Para contextualizar esto, y para excluir la posibilidad de que existiera un generalizado incremento diplomático para el primer semestre de cada año, contemplaremos los fondos normeseteños más nutridos, es decir los de Cardeńa, Sahagún y León ${ }^{35}$. Observamos que, aunque existe una ligera predominancia de actividad diplomática en el primer semestre de cada ańo (c 56\%), no es comparable con la explosión de actividad que observamos a principios de 950 .

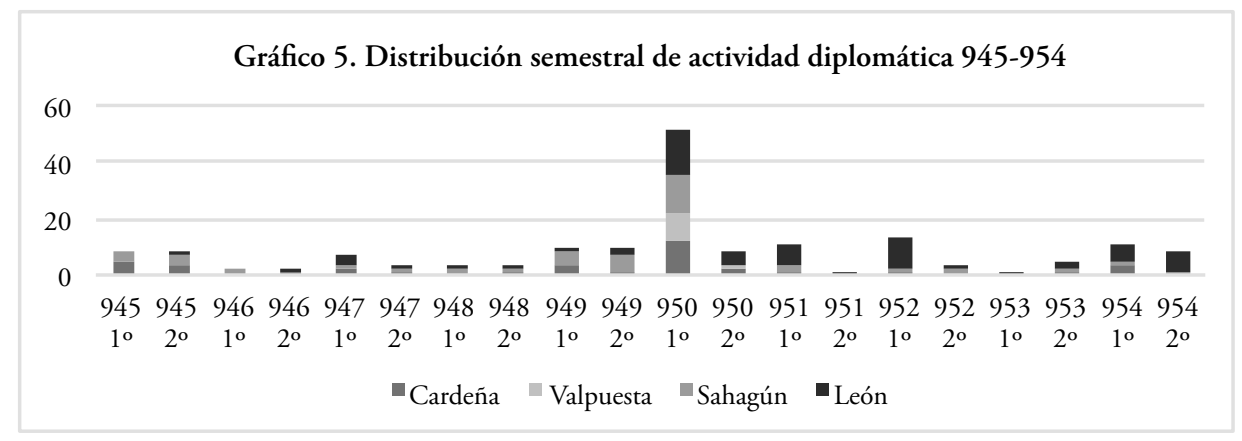

Lo que postulamos es que una parte importante de esta abundancia diplomática del año 950 es consecuencia del incendio catastrófico que se ha analizado en la primera parte del trabajo. Si, aunque sea con matizaciones, resulta sostenible esta hipótesis, nos proporcionaría una valiosa herramienta de análisis tanto de la economía campesina, sobre todo desde la perspectiva de su precariedad, como de las dinámicas, ritmos y motivos de la producción diplomática. Desde la emergencia de la Nueva Historia es abundante la historiografía que emplea las transacciones campesinas como herramienta para el análisis de la economía rural altomedieval, tanto para la Europa occidental en general ${ }^{36}$ como

$35 \mathrm{Al}$ margen del dosier de Buezo, el fondo valpositano es cuantitativamente inadecuado para este tipo de análisis. Entre 930 y 969 (excluyendo 950), en las otras tres colecciones analizadas observamos:

- en Cardeña, primer semestre 58 (55\%), segundo semestre 47 (45\%);

- en Sahagún, primer semestre 104 (53\%), segundo semestre 94 (47\%);

- en León, primer semestre 162 (60\%), segundo semestre 109 (40\%);

- en conjunto, primer semestre 324 (56\%), segundo semestre 250 (44\%).

36 Para ofrecer algunos ejemplos destacados, y sin ningún ánimo de exhaustividad: DuBy, Georges. Economia rural y vida campesina en el Occidente medieval. Barcelona: Península, 1968 [1962]; RosenwEIN, Barbara H. «Property transfers and the Church, eighth to eleventh Centuries. An overview». Mélanges de l'École Française de Rome. Moyen-Âge, 1999, vol. 111, n. ${ }^{\circ}$, pp. 563-575, que sirve como introducción a todo este volumen de estudios dedicados a la transferencia de patrimonio en la Europa altomedieval; Feller, Laurent y Wickham, Chris (eds.). Le marché de la terre au Moyen Âge. Roma: École Française de Rome, 2005. 
EL GRAN INCENDIO CASTELLANO DE 949. HUELLA DIPLOMÁTICA Y MEMORIA HISTÓRICA DE UN DESASTRE NATURAL

para la Meseta $\operatorname{Norte}^{37}$. Notamos, no obstante, que la hiperproducción diplomática que observamos no se comenta en los citados estudios monográficos dedicados a dos de estas instituciones (Cardeña y Sahagún), puesto que estos pretenden identificar tendencias más amplias que las dinámicas anuales que analizamos aquí. Mínguez, por ejemplo, al estudiar los ciclos diacrónicos de adquisiciones de Sahagún, agrupaba las transacciones en bloques de cinco años, para así evitar la distracción de anomalías estadísticas. Siguiendo esta metodología, el periodo 950-954 no destaca en absoluto en cuanto al volumen de transacciones, aunque sí ligeramente con referencia a la relativa modestia de estas, algo que concuerda con lo que observamos nosotros ${ }^{38}$. Por lo tanto, con respecto a esta tradición historiográfica, este caso tan específico supondría una aportación algo diferente, al analizarse la economía campesina familiar sujeta a una prueba de estrés muy concreta.

Es importante matizar que no debemos pensar que toda esta actividad diplomática de 950 fuera necesariamente consecuencia del incendio. Sería lógico que hubiera también actividad ajena a la dinámica que nos interesa, y en reconocimiento de esto ya hemos excluido del análisis los textos de clara factura no campesina. Pero también es posible, incluso probable, que entre el pretendido corpus campesino restante haya textos cuyo origen se debe a dinámicas ajenas a la que nos interesa. El ejemplo más claramente diferenciable es Cardeña74, que recoge una donación en Esgueva (una comarca meridional alejada de la geografía del incendio) cuya generosidad -una iglesia- contrasta con la modestia de la mayoría de las transacciones de aquel año. De modo parecido, podríamos cuestionar la pertinencia a la dinámica que nos interesa de la venta (en sí atípica entre las transacciones de Cardeña) relativamente amplia que hizo el matrimonio Falcón y Anderquina en Rama, de nuevo un núcleo algo alejado, en este caso más al norte, de la mayoría de estas transacciones (Cardeña67). Por su parte, en la documentación leonesa la venta por Fortunio y María de su villa en Ardón por 50 sueldos destaca por encima de las cifras manejadas en otras compraventas, que nunca rebasan los 18 sueldos.

En cambio, con referencia a la calidad social de los protagonistas de nuestros textos no se aprecian diferencias, más allá de la anteposición en tres casos leoneses de doña al nombre de la esposa, pero nunca con el equivalente masculino, lo cual me inclina a pensar que se debe más a una convención onomástica que a un auténtico marcador de rango social. Por lo demás, carecemos de indicios, al margen de la escala de algunas enajenaciones mencionadas arriba, de que estos donantes y vendedores fuesen otra cosa

37 En España los primeros trabajos de esta índole partieron desde la perspectiva de los grandes señoríos monásticos: GARCía DE CoRTÁZAR, José Ángel. El dominio del monasterio de San Millán de la Cogolla (siglos X a XIII). Introducción a la historia rural de Castilla altomedieval. Salamanca: Ediciones Universidad de Salamanca, 1969; Moreta Velayos, Salustiano. El monasterio de San Pedro de Cardeña. Salamanca: Ediciones Universidad de Salamanca, 1971; Mínguez Fernández, José María. El dominio del monasterio de Sahagún en el siglo $X$. Salamanca: Ediciones Universidad de Salamanca, 1980. Después y paulatinamente los propios campesinos han ido ganando protagonismo, con referencia a las comunidades aldeanas (Álvarez Borge, Ignacio (ed.). Comunidades locales y poderes feudales en la Edad Media. Logroño: Universidad de La Rioja, 2001), desde el exiguo registro material (Quirós Castillo, Juan Antonio (coord.). Arqueología del campesinado medieval: la aldea de Zaballa. [Bilbao]: Universidad del País Vasco, 2012), o recientemente con un retorno al análisis pormenorizado de la diplomática (Davies, Acts of Giving, 2007; Portass, Robert. The Village World of Early Medieval Northern Spain. Woodbridge: Boydell, 2017).

38 Mínguez Fernández, El dominio, pp. 207-215, y sobre todo los gráficos 1 y 2 en la p. 214. 
EL GRAN INCENDIO CASTELLANO DE 949. HUELLA DIPLOMÁTICA

Y MEMORIA HISTÓRICA DE UN DESASTRE NATURAL

DAVID PETERSON

que miembros del campesinado más o menos próspero. Podemos añadir que en algunos casos (Sahagún121, Sahagún125) sabemos que los bienes vendidos habían sido comprados anteriormente, lo cual sugiere un cambio -quizás repentino, aunque esto no lo podemos saber- en la fortuna del vendedor.

Con respecto a los destinatarios y a los tipos de transacción, en cambio, sí observamos diferencias: mientras en Castilla son arquetípicamente los monasterios los receptores de las donaciones, en León son compradores diversos, tanto instituciones como particulares. Este es quizás el aspecto más problemático para la consideración del conjunto de transacciones como un corpus homogéneo por lo que tendríamos que contemplar la posibilidad de que los casos castellanos y los leoneses obedezcan a dinámicas diferentes. No obstante, seguiría llamando la atención el hecho de que el año 950 fuera el de máxima actividad diplomática en ambos escenarios. Más probable me parece que el tipo de institución receptora condicionara el tipo de transacción y, por tanto, lo que vemos es una diferencia regional en las formas elegidas para solventar esencialmente la misma problemática.

Aunque todos estos documentos, así como los bienes cuya adquisición detallan, acabarían en manos de las cuatro instituciones (Valpuesta, Cardeńa, Sahagún y León), la pluralidad de los destinatarios en primera estancia es notable, siendo la excepción el dosier de Buezo. Pues, en la documentación de Cardeña, además del monasterio en sí, vemos cómo el por lo demás desconocido cenobio de San Andrés de Villalbilla recibe tres donaciones ese año, mientras que los particulares Anderazo y el presbítero Félix realizan compras. En la documentación leonesa, donde la compraventa es la forma predominante de adquisición, los compradores fueron diversos pues, además del obispo leonés y el abad sahagunés, figuran otros monasterios (Abellar, San Miguel, Valdevimbre), el prolífico Iscam de Melgar, los presbíteros Citayo (con su hermana Filauria), Julián y Melic, así como numerosos particulares. Pero lejos de ser problemática, esta diversificación de agentes adquisidores refuerza la impresión de que estamos ante una dinámica plural y, por tanto, ajena a la práctica archivística o al proyecto adquisitivo de una institución u otra. Una excepción en este último sentido podría ser la actividad del mencionado Iscam, con mucho el adquisidor más prolífico, que no se limita a 950 (Sahagún94a, Sahagún162, Sahagún164, etc.), pero de nuevo es este el año que destaca como el más productivo en todo su periodo de actividad adquisidora.

En Castilla, como hemos comentado, las donaciones son las transacciones que dominan, e incluso entre ellas se emplean diferentes fórmulas distintivas: una donación sin más; la donación corpus et anima, según la cual se incorporaba un individuo (o grupo) en una comunidad religiosa aportando sus bienes; la donación post obitum; o la profiliación. Generalmente, no se explicitan ni los motivos ni las circunstancias que están detrás de la donación, siendo el silencio la tónica habitual en este tipo de transacción campesina ${ }^{39}$.

39 Davies, Acts of Giving, p. 108. La bibliografía sobre la motivación memorística para las donaciones altomedievales es extensa: DuBy, Economia rural y vida campesina, pp. 80-81; y, centrándonos en el espacio que nos ocupa, Moreta Velayos, El monasterio de San Pedro de Cardeña, pp. 101-105; Davies, Acts of Giving, pp. 30-34; Fernández Conde, Francisco Javier. "La religiosidad medieval y las donaciones a la Iglesia». En García Leal, Alfonso (ed.). Las donaciones piadosas en el mundo medieval. Oviedo: Universidad de Oviedo, 2012, pp. 9-58. 
EL GRAN INCENDIO CASTELLANO DE 949. HUELLA DIPLOMÁTICA Y MEMORIA HISTÓRICA DE UN DESASTRE NATURAL DAVID PETERSON

Aunque el contraste entre el tipo de transacción favorecido en los dos espacios es llamativo, Davies ya comentó que la distinción entre la donación y la compraventa no siempre es clara en la documentación de la Meseta Norte en el siglo X, y que las supuestas donaciones frecuentemente contienen un 'aire de reciprocidad'40. Si añadimos la posibilidad de que efectivamente fuesen las instituciones quienes determinasen la fórmula aplicada, quizás el contraste sea más ilusorio que real.

TABLA 5. Grupos familiares protagonistas de las transacciones campesinas de 950.

\begin{tabular}{|l|c|c|c|c|c|c|}
\hline & VALPUESTA & CARDEÑA & SAHAGÚN & LEÓN & TOTAL & $\%$ \\
\hline mujer & & 1 & 1 & 2 & 4 & 6,7 \\
\hline madre e hijos & 1 & 1 & 2 & 3 & 7 & 11,7 \\
\hline varón & 1 & 6 & 3 & 1 & 11 & 18,3 \\
\hline padre e hijos & 2 & & & 1 & 3 & 5,0 \\
\hline matrimonio & 7 & 3 & 5 & 10 & 25 & 41,7 \\
\hline otros & 1 & 3 & 3 & 3 & 10 & 16,7 \\
\hline Total & 12 & 14 & 14 & 20 & 60 & \\
\hline
\end{tabular}

En su estudio de donaciones del norte de Iberia en el siglo x, Davies nota que el 46\% de los enajenadores eran hombres individuales, mientras que los matrimonios suponían el $29 \%{ }^{41}$. Aunque evidentemente el muestreo es mucho menor para el ańo 950, las proporciones son diferentes (Tabla 5): los matrimonios suponen ya el 40\%, además de lo que parece ser una alta incidencia de 'padres solteros', que interpretamos como viudas -madres con hijos sin referencia al padre- y viudos, padres con hijos sin mención de la madre. Las mujeres, infrarrepresentadas en la mayoría de registros altomedievales, aquí tienen casi el mismo protagonismo que los hombres y el doble en los casos de presumible viudedad. Parece desproporcionadamente alto el número de grupos familiares mermados que buscan apoyo -de instituciones o simplemente de vecinos más acomodados- en momentos de crisis $^{42}$. Incluso en las transacciones protagonizadas por matrimonios o individuos, y cuando a priori no hay indicios de desestructuración familiar, las circunstancias concretas recogidas en algunas de estas actas apuntan hacia grupos familiares también disfuncionales ${ }^{43}$ :

- El matrimonio Paterno \& Anderazo entrega su único hijo al monasterio de Buezo (Valpuesta21);

- Anderquina \& Nuño, respectivamente tía y sobrino, recuerdan al difunto hermano de ella, padre de él (Valpuesta27);

40 Davies, Acts of Giving, p. 114, 136, 156 y 159.

41 Davies, Acts of Giving, p. 172.

42 "The relatively large proportion of gifts from couples and single women (sometimes explicitly widowed) must make it very likely that many of these otherwise-unexplained formulas conceal protection arrangements", Davies, Acts of Giving, p. 59.

43 Sobre el predominio de la familia conyugal en las transacciones del periodo, en contraste con lo observado en la documentación de 950, cf. PAstor Díaz De Garayo, Ernesto. Castilla en el tránsito de la Antigüedad al feudalismo: poblamiento, poder politico y estructura social del Arlanza al Duero (siglos VII-XI). Valladolid: Junta de Castilla y León, 1996, pp. 298-299. 
EL GRAN INCENDIO CASTELLANO DE 949. HUELLA DIPLOMÁTICA Y MEMORIA HISTÓRICA DE UN DESASTRE NATURAL DAVID PETERSON

- Eulalia, encontrándose enferma, dona post mortem al monasterio de Abellar la corte en la ciudad de León en la cual vive, añadiendo que lo hace en memoria de sus hijos difuntos, Álvaro, Eufimia y Filicia (León230);

- Gesmira explica que su donación al matrimonio Flaino y Brunildi es en agradecimiento por los cuidados recibidos en la casa de la pareja (León222).

En otra ocasión disponemos de un relato singularmente gráfico que nos acerca a la dinámica de desesperación detrás de una de las donaciones valpositanas, y que además aporta un contexto cronológico muy preciso. Se trata de la explicación que nos ofrece Sanzone por su donación al monasterio de Buezo el 1 de junio de 950:

Que sea conocido por todos los hombres el bien y esencia piadosa que me hicisteis este año nefasto, [todo] por la misericordia honrosa y la salvación de vuestra alma. Yo yacía en vuestra puerta desecho e hinchado por el hambre, y no me fiaba en mi alma de vivir un solo día más sobre la tierra; me moría de hambre [para que] mi hijo comiera pan. Y Dios inspiró en vosotros el buen espíritu y por vuestra honrosa misericordia os apiadasteis de mi aquel conocido calendas [10․ de junio, y [así] apartasteis para mí la ración de comida de uno de vosotros, es decir, el régimen de pan de todos los días, para que lo comiera como un compañero más, y además una cabra con leche, con la cual revivió mi hija, y arropasteis mi cuerpo con una saya y un manto, lo cual me hizo bien; por esto mi alma desconfiaba de seguir en esta vida, desde el día del calendas de junio hasta el día de san Cristóbal (Valpuesta28, 1 de junio de 950, traducción propia $\left.{ }^{44}\right)$.

Este estremecedor relato es el que llamó la atención a Peña Pérez, quien lo entendía en términos genéricos de una mala cosecha ${ }^{45}$, pero que ahora lo podemos contextualizar mejor. La coincidencia entre la frase empleada aquí, anno pessimo, y el anno iniquo de los Anales Castellanos Segundos es llamativa, pero, extrañamente, a pesar de tan larga exposición, Sanzone no explicita la causa de la hambruna, aunque sí la cronología del proceso. El documento se fecha el 1 (calendas) de junio de 950, pero en el relato que narra hechos necesariamente anteriores a tal fecha se explicita otras dos veces la misma fecha y la implicación es clara: se refiere al año inmediatamente anterior (isto anno pessimo) y más específicamente al notorio (notum) primer día de junio. Exactamente un año después, ya recuperado, Sanzone está agradeciendo la ayuda que el monasterio le prestó en aquel momento. Notamos que el otro día mencionado, San Cristóbal, es el 10 de julio: durante 40 días, por lo tanto, el monasterio le había cuidado; un periodo de tiempo

44 Notum sit homnibus hominibus beneficium et uiscera pietatjs quod inpendistis super me in isto anno pessimo, per uestra digna misericordia et remedium anime uestre. Iacebam ad ianua uestra defectus et inflatus de famen et non abiam ego fiducia de anima mea que bibissem uno die super terram, manducando filius meus panem ego morieba de famen. Et inspirabit Deus in uos spiritus bonus et in pietatis super me pro uestra digna misericordia in die notum kalendas iunias et separastis mici ipsam rationem quod unus ex uobis manducabat, id est, regula panis de die in die et ipsum manducare quod et uos et ipso conpanio, et super hoc capra cum lacte, unde revibesci filia mea, et induististis corpus meum de saia et manto, quod mici bene fuit, unde non abui fiducia de anima mea que fuissem iam in ista luce, de die kalendas iunias usque in diem Sancti Christofori (Valpuesta28, 1 de junio de 950; transcripción de Ruiz Asencio et álii).

45 Peña Pérez, «Los espacios sociales en la España cotidiana», pp. 306-307. 
EL GRAN INCENDIO CASTELLANO DE 949. HUELLA DIPLOMÁTICA Y MEMORIA HISTÓRICA DE UN DESASTRE NATURAL

sospechosamente bíblico, pero aquí expresado en términos muy precisos que sugieren verosimilitud. El 1 de junio (de 949), recordemos, era el día exacto en que, tanto según el Cronicón Burguense como el Cronicón de Cardeña, a la novena hora se desató el incendio. Asimismo, notamos que dos de las donaciones cardenienses se hacen igualmente en el aniversario o su víspera: Cardeña76, el 31 de mayo; Cardeña77, el propio 1 de junio.

La pregunta ineludible es por qué un incendio se iba a traducir en un incremento de actividad diplomática y, además, por qué aparece el pico de actividad en el primer semestre de 950 en vez de en los meses inmediatamente posteriores al incendio de junio 949. Debemos pensar en términos de unas personas de recursos limitados que en tiempos de crisis buscan ayuda en una institución o familia más o menos cercanas. La ayuda puede ser inmediata, como en el caso de Sanzone, y caridad coyuntural de este tipo no tendría por qué generar documentación. En cambio, el periodo más duro del año agrícola se da hacia el final del invierno, cuando los escasos recursos disponibles se han ido gastando. Es en este periodo, ya a principios de 950, cuando diferentes unidades familiares, muchas de ellas echando en falta familiares fundamentales para la productividad eficaz de la pequeña explotación, buscan ayuda, en algunos casos vendiendo una modesta propiedad a cambio de sustento, y en otros entregándose con todo su exiguo patrimonio al cenobio $^{46}$. Ya en plena primavera observamos otra dinámica-menos importante en términos cuantitativos, aunque de singular valor en el caso del testimonio de Sanzone- cuando se recuerda y se agradece la ayuda puntual prestada el año anterior. Este es también el periodo cuando se concentran las pocas donaciones en Sahagún y León, en contraste con las ventas que habían dominado antes. Las diferentes circunstancias familiares y los ritmos del año agrícola explicarían así los diferentes tipos de documento generados, pero casi todos ellos tienen su origen en la penuria de las familias campesinas después de un año nefasto. Otro indicio de ello es la modestia de la mayoría de estas transacciones, que repetidamente especifican el número preciso de frutales donados e incorporan referencias a fracciones de bienes ${ }^{47}$.

El incendio de 949 fue extraordinario en varios sentidos. Por su aparición en la analística castellana como uno de los escasísimos sucesos no geopolíticos documentados y, también, como el único hecho del siglo $\mathrm{x}$ recordado en ambas tradiciones analísticas castellanas. Además, en los Anales Castellanos Terceros, se le dedica una entrada mucho más desarrollada que lo habitual para las noticias del siglo $\mathrm{x}$. Es extraordinario también el haber dejado huella en ambos registros documentales principales, tanto la analística como la diplomática. Su huella diplomática nos acerca de manera pormenorizada

46 Sobre el modelo de pequeña explotación familiar y la problemática de «situaciones de deficiente capacitación laboral», cf. García González, Juan José y Fernández de Mata, Ignacio. «En el corazón de las comunidades locales. La pequeña explotación agropecuaria familiar de la cuenca del Duero en la transición de la Antigüedad a la Edad Media». En Álvarez Borge (coord.), Comunidades locales y poderes feudales en la Edad Media, pp. 203-254, especialmente p. 222.

47 Una vińa, un huerto y una herrén (Valpuesta21); un campo de cereal (Valpuesta23); una faza con cuatro manzanos, un peral, doce manzanos y un fresno, todo a cambio de un carnero (Valpuesta24); una parte de un huerto (Valpuesta27); un campo de cereal y un huerto con cinco manzanos, tres nogales y un peral (Valpuesta28); un huerto con dos manzanos, los pomares y la cuarta parte de un campo de cereal (Valpuesta29). 
EL GRAN INCENDIO CASTELLANO DE 949. HUELLA DIPLOMÁTICA

Y MEMORIA HISTÓRICA DE UN DESASTRE NATURAL

DAVID PETERSON

(aunque evidentemente fragmentaria e incompleta) a las consecuencias humanas de un desastre natural y a la relación entre tal coyuntura y la producción de actas notariales. Y, en tercer lugar, parece haber sido extraordinaria la envergadura del desastre, tanto por su descripción analística como por su huella documental. Teniendo todo esto en cuenta, resulta sorprendente que un hecho tan singular y tan bien documentado haya estado cerca del absoluto destierro historiográfico. Tampoco es que su excepcionalidad le reste relevancia histórica consignándola al anecdotario, pues la crisis que retrata nos permite apreciar ciertas grietas estructurales en la precaria economía campesina y nos acerca de manera excepcional a los campesinos más vulnerables en un periodo cuando la mayoría de la documentación todavía emana de las clases pudientes.

Adjuntamos a continuación una relación de los 60 documentos del año 950 que constituyen un singular corpus de inusitada actividad campesina en la Meseta Norte. Es probable que no todas estas transacciones deban entenderse en el contexto del incendio de 949, pero creemos que la gran mayoría sí. Este corpus sincrónico, contextualizado como ningún otro por la confluencia entre la analística y la diplomática, nos brinda una oportunidad excepcional para estudiar las consecuencias de un desastre natural en la sociedad rural altomedieval. Aun falta análisis por hacer sobre este corpus, tarea que escapa de las dimensiones y pretensiones de este trabajo, pues lo que se ha pretendido aquí es la identificación del conjunto de estas modestas transacciones y demostrar su relación con los acontecimientos de 949 recordados en la analística. Asimismo, son interesantes las implicaciones para nuestra comprensión de las dinámicas de generación y conservación documental. Pues, lo que se observa aquí parece ser una relación directa entre un acontecimiento concreto y la cuantía de documentación conservada en diferentes instituciones, y esto sugiere que la proporción de documentación medieval que ha sobrevivido esté más estrechamente relacionada con la que se generó en el primer momento de lo que se ha sugerido. Este aspecto es más llamativo aun si tenemos en cuenta la modestia de la mayor parte de estas transacciones, a pesar de la cual han sido copiadas y así preservadas en los cartularios dejándonos un estrato de ceniza documental como testigo del incendio de 949.

\section{REFERENCIAS BIBLIOGRÁFICAS}

ÁldVAREZ Borge, Ignacio (ed.). Comunidades locales y poderes feudales en la Edad Media. Logroño: Universidad de La Rioja, 2001.

Álvarez Martínez, Ursicino. Historia general de la provincia de Zamora. Zamora: La Seña Bermeja, 1889.

Becerro Galicano Digital (www.ehu.eus/galicano - consultado 03/08/2017).

Calvo Madroño, Ismael. Descripción geográfica, histórica y estadística de la provincia de Zamora. Madrid: Imprenta de Victoriano Suárez, 1914.

Davies, Wendy. Acts of Giving. Individual, Community, and Church in Tenth-Century Christian Spain. Oxford: Oxford University Press, 2007.

Duby, Georges. Economia rural y vida campesina en el Occidente medieval. Barcelona: Península, 1968 [1962]. 
EL GRAN INCENDIO CASTELLANO DE 949. HUELLA DIPLOMÁTICA Y MEMORIA HISTÓRICA DE UN DESASTRE NATURAL

Feller, Laurent y Wiскнам, Chris (eds.). Le marché de la terre au Moyen Âge. Roma: École Française de Rome, 2005.

Fernández Conde, Francisco Javier. «La religiosidad medieval y las donaciones a la Iglesia». En García Leal, Alfonso (ed.). Las donaciones piadosas en el mundo medieval. Oviedo: Universidad de Oviedo, 2012, pp. 9-58.

Fernández Duro, Cesáreo. Memorias históricas de la ciudad de Zamora. Madrid: Sucesores de Rivadeneyra, 1882.

Flórez, Henrique. España Sagrada: theatro geographico-historico de la iglesia de España, vol. XXIII (de la iglesia de Tuy). Madrid: Antonio Marín, 1767.

Galbis Rodríguez, José. Catálogo sísmico de la zona comprendida entre los meridianos $5^{\circ}$ E y $20^{\circ}$ Wy paralelos $45^{\circ}$ y $25^{\circ} \mathrm{N}$. Madrid: Instituto Geográfico, Catastral y de Estadística, 1932.

García de Cortázar, José Ángel. El dominio del monasterio de San Millán de la Cogolla (siglos X a XIII). Introducción a la historia rural de Castilla altomedieval. Salamanca: Ediciones Universidad de Salamanca, 1969.

García GonzÁlez, Juan José y Fernández de Mata, Ignacio. «En el corazón de las comunidades locales. La pequeña explotación agropecuaria familiar de la cuenca del Duero en la transición de la Antigüedad a la Edad Media». En Álvarez Borge, Ignacio (ed.). Comunidades locales y poderes feudales en la Edad Media. Logroño: Universidad de La Rioja, 2001, pp. 203-254.

Gómez-Moreno, Manuel. "Anales castellanos». En Discursos leídos ante la Real Academia de la Historia. Madrid: Real Academia de la Historia, 1917.

Gonzalo de Berceo. Vida de San Millán de la Cogolla. Edición y comentario, ed. Brian Dutton. Madrid: Espasa-Calpe, 1992.

Martín Iglesias, José Carlos. «Los Annales Castellani Antiquiores y Annales Castellani Recentiores: edicion y traducción anotada». Territorio, Sociedad y Poder, 2009, vol. 4, pp. 203-226.

Martínez DíEz, Gonzalo. "Tres anales burgaleses medievales». Boletín del Instituto Fernán González, 2004, vol. 83, pp. 227-263.

Martínez Díez, Gonzalo. Colección documental del Monasterio de San Pedro de Cardeña. Burgos: Caja Círculo, 1998.

Martínez Díez, Gonzalo. El condado de Castilla. (711-1038). La Historia frente a la Leyenda. Valladolid-Madrid: Junta de Castilla y León-Marcial Pons, 2005.

Martinez Solares, José Manuel. «Sismicidad histórica de la Península Ibérica». Física de la Tierra, 2003, vol. 15, pp. 13-28.

Martínez Solares, José Manuel y Mezcua, Julio. Catálogo sísmico de la Península Ibérica (880 a. C.-1900). Madrid: Dirección General del Instituto Geográfico Nacional, 2002.

Mezcua, Julio y Martínez Solares, José Manuel. Sismicidad del área iberomogrebi. Madrid: Instituto Geográfico Nacional, 1983.

Mínguez Fernández, José María. Colección diplomática del Monasterio de Sahagún, (siglos IX y X). León: Centro de Estudios e Investigación San Isidoro, 1976.

Mínguez Fernández, José María. El dominio del monasterio de Sahagún en el siglo X. Salamanca: Ediciones Universidad de Salamanca, 1980.

Moreta Velayos, Salustiano. El monasterio de San Pedro de Cardeña. Salamanca: Ediciones Universidad de Salamanca, 1971.

Munuera Quiñonero, José María. A study of seismicity on the Península Ibérica area. Technical note $n .1$ «Seismic Data». Madrid: Instituto Geográfico y Catastral, 1963.

Nelson, Jinty. The Annals of St-Bertin. Manchester: Manchester University Press, 1991.

Pastor Díaz de Garayo, Ernesto. Castilla en el tránsito de la Antigüedad al feudalismo: poblamiento, poder politico y estructura social del Arlanza al Duero (siglos VII-XI). Valladolid: Junta de Castilla y León, 1996. 
EL GRAN INCENDIO CASTELLANO DE 949. HUELLA DIPLOMÁTICA Y MEMORIA HISTÓRICA DE UN DESASTRE NATURAL DAVID PETERSON

Peña Pérez, Francisco Javier. «Los espacios sociales en la España cotidiana. Valpuesta en la Edad Media». En MCC Aniversario del obispado de Valpuesta: 804-2004. Miranda de Ebro: Fundación Cultural Miranda de Ebro, 2004, pp. 291-319.

Pérez de Urbel, Justo. El condado de Castilla. Madrid: Siglo Ilustrado, 1969-1970, 3 vols.

Poema de Fernán González, ed. Emilio Alarcos Llorach. Madrid: Castalia, 1965.

Portass, Robert. The Village World of Early Medieval Northern Spain. Woodbridge: Boydell, 2017.

Puig y Larraz, Gabriel. Descripción física y geológica de la provincia de Zamora. Madrid: Manuel Tello, 1883.

Pyne, Stephen J. Fire in America. A Cultural History of Wildland and Rural Fire: Princeton: Princeton University Press, 1982.

Quirós Castillo, Juan Antonio (coord.). Arqueología del campesinado medieval: la aldea de Zaballa. [Bilbao]: Universidad del País Vasco, 2012.

Ramos Remedios, Emiliana. "Francos y vascos en el norte de Castilla (IX-XIII): los cambios en las denominaciones personales». Nouvelle Revue d'Onomastique, 2014, vol. 56, pp. 197-225.

Reuter, Timothy. The Annals of Fulda. Manchester: Manchester University Press, 1992.

Rosenwein, Barbara H. «Property transfers and the Church, eighth to eleventh Centuries. An overview». Mélanges de l'École Française de Rome. Moyen-Âge, 1999, vol. 111, n. ${ }^{\circ}$ 2, pp. 563-575.

Ruiz Asencio, José Manuel. «Los cartularios de Valpuesta». En MCC Aniversario del obispado de Valpuesta: 804-2004. Miranda de Ebro: Fundación Cultural Miranda de Ebro, 2004, pp. 354-381.

Ruiz Asencio, José Manuel; Ruiz Albi, Irene y Herrero Jiménez, Mauricio. Los Becerros Gótico y Galicano de Valpuesta. Burgos: Instituto Castellano y Leonés de la Lengua, 2010, 2 vols.

Sáez, Emilio y SÁez, Carlos. Colección diplomática del monasterio de Celanova (842-1230). Alcalá de Henares: Universidad de Alcalá, 2000.

SÁez SÁnchez, Emilio. Colección documental del Archivo de la Catedral de León (775-1230). León: Centro de Estudios e Investigación San Isidoro, 1987-1991.

WRIGHT, Roger. «How Scribes Wrote Ibero Romance Before Ibero Romance was Invented», https://liverpool.academia.edu/RogerWright.

Zabalza Conde, Manuel. «Tipología de los documentos de Valpuesta». En MCC Aniversario del obispado de Valpuesta: 804-2004. Miranda de Ebro: Fundación Cultural Miranda de Ebro, 2004, pp. 320-353.

\section{Anexos}

Anexo 1: Documentación normeseteña, 930-970.

\begin{tabular}{|c|c|c|c|c|}
\hline Año & LeóN & SAHAGúN & CARDEŃa & VALPUeSta \\
\hline 930 & 2 & 6 & 0 & 0 \\
\hline 931 & 2 & 1 & 2 & 0 \\
\hline 932 & 4 & 8 & 4 & 0 \\
\hline 933 & 2 & 5 & 0 & 0 \\
\hline 934 & 2 & 3 & 0 & 0 \\
\hline 935 & 3 & 1 & 1 & 1 \\
\hline 936 & 6 & 5 & 1 & 0 \\
\hline 937 & 12 & 9 & 2 & 0 \\
\hline
\end{tabular}

(C) Ediciones Universidad de Salamanca

Stud. hist., H. ${ }^{a}$ mediev., 37(1), 2019, pp. 139-164 
EL GRAN INCENDIO CASTELLANO DE 949. HUELLA DIPLOMÁTICA

162

Y MEMORIA HISTÓRICA DE UN DESASTRE NATURAL

DAVID PETERSON

\begin{tabular}{|c|c|c|c|c|}
\hline Año & LeÓN & SAHAGÚN & Cardeña & Valpuesta \\
\hline 938 & 3 & 3 & 0 & 0 \\
\hline 939 & 6 & 2 & 2 & 2 \\
\hline 940 & 4 & 1 & 1 & 1 \\
\hline 941 & 8 & 5 & 2 & 0 \\
\hline 942 & 10 & 2 & 3 & 0 \\
\hline 943 & 18 & 9 & 3 & 0 \\
\hline 944 & 10 & 1 & 2 & 0 \\
\hline 945 & 2 & 8 & 9 & 1 \\
\hline 946 & 1 & 2 & 1 & 1 \\
\hline 947 & 5 & 2 & 3 & 0 \\
\hline 948 & 5 & 3 & 1 & 0 \\
\hline 949 & 5 & 10 & 5 & 0 \\
\hline 950 & 22 & 14 & 14 & 12 \\
\hline 951 & 10 & 7 & 1 & 0 \\
\hline 952 & 19 & 3 & 1 & 1 \\
\hline 953 & 5 & 1 & 1 & 0 \\
\hline 954 & 18 & 5 & 3 & 0 \\
\hline 955 & 8 & 6 & 1 & 0 \\
\hline 956 & 7 & 3 & 2 & 1 \\
\hline 957 & 1 & 4 & 3 & 2 \\
\hline 958 & 5 & 4 & 2 & 0 \\
\hline 959 & 14 & 9 & 3 & 0 \\
\hline 960 & 12 & 11 & 0 & 0 \\
\hline 961 & 16 & 11 & 2 & 0 \\
\hline 962 & 7 & 12 & 8 & 1 \\
\hline 963 & 11 & 10 & 5 & 1 \\
\hline 964 & 12 & 6 & 7 & 0 \\
\hline 965 & 11 & 18 & 9 & 0 \\
\hline 966 & 6 & 4 & 2 & 1 \\
\hline 967 & 5 & 6 & 1 & 1 \\
\hline 968 & 4 & 0 & 4 & 2 \\
\hline 969 & 1 & 2 & 6 & 0 \\
\hline Total & 304 & 222 & 117 & 28 \\
\hline Media anual & 7,6 & 5,55 & 2,93 & 0,7 \\
\hline
\end{tabular}

Anexo 2. La documentación de 950.

\begin{tabular}{|l|r|l|l|l|l|l|}
\hline Doc & Date & \multicolumn{1}{|c|}{ Núcleo } & & \multicolumn{1}{|c|}{ Grupo } & \multicolumn{1}{c|}{ Tipo } & \multicolumn{1}{c|}{ Adquiere } \\
\hline V20 & $25 / 02$ & Buezo & Tello Muñoz \& Vandilo & X & D & Buezo \\
\hline V21 & $18 / 03$ & Buezo & Paterno \& Anderazo & X & D & Buezo \\
\hline V22 & $18 / 03$ & Buezo & Siseberto & IV & D & Buezo \\
\hline V23 & $25 / 03$ & Buezo & Placenti \& Muñata & X & D & Buezo \\
\hline V24 & $29 / 03$ & Buezo & Ciezo \& Flaina & X & V & Buezo \\
\hline
\end{tabular}

(C) Ediciones Universidad de Salamanca

Stud. hist., H. ${ }^{\mathrm{a}}$ mediev., 37(1), 2019, pp. 139-164 
EL GRAN INCENDIO CASTELLANO DE 949. HUELLA DIPLOMÁTICA

Y MEMORIA HISTÓRICA DE UN DESASTRE NATURAL

DAVID PETERSON

\begin{tabular}{|c|c|c|c|c|c|c|}
\hline Doc & DATE & NúCLEO & ENAJENA & Grupo & Tipo & AdQUiere \\
\hline V25 & $04 / 04$ & Buezo & Ciezo \& Flaina & $\mathrm{X} \& \mathrm{H}$ & $\mathrm{D}$ & Buezo \\
\hline V26 & $17 / 04$ & Buezo & Vela y su hijo Placenti & $\mathrm{P} \& \mathrm{H}$ & $\mathrm{D}$ & Buezo \\
\hline V27 & $? / 04$ & Buezo & Anderquina \& Nuño & Tía \& sobrino & $\mathrm{V} / \mathrm{D}$ & Buezo \\
\hline V28 & $01 / 06$ & Buezo & Sanzone & $\mathrm{P} \& \mathrm{H}$ & $\mathrm{D}$ & Buezo \\
\hline V29 & $10 / 06$ & Buezo & Bona e hijo Velasco & $\mathrm{M} \& \mathrm{H}$ & V & Buezo \\
\hline V30 & $18 / 08$ & Buezo & Juan \& Cara & $X$ & D & Buezo \\
\hline V31 & $? ? \mid ? ?$ & Buezo & Fruela \& María & $X$ & $\mathrm{D}$ & Buezo \\
\hline C67 & $01 / 01$ & Rama & Falcón \& Anderquina & $X$ & V & Cardeńa \\
\hline C68 & $22 / 01$ & Marmellar & Severo y Esteban & socios & DA\&C & Cardeña \\
\hline C69 & $23 / 01$ & Canaleja & Elias \& Ogoa & $\mathrm{X}$ & V & Anderazo \\
\hline $\mathrm{C} 70$ & $01 / 02$ & Villalbilla & Ositia & IM & DPf & Villalbilla \\
\hline $\mathrm{C} 71$ & $01 / 03$ & Cardeńadijo & Rapinato & IV & DA\&C & Cardeńa \\
\hline $\mathrm{C} 72$ & $12 / 04$ & Carcedo & Egilo \& Goto & $\mathrm{Hh}$ & $\mathrm{DA} \& \mathrm{C}$ & Cardeña \\
\hline $\mathrm{C} 73$ & $28 / 04$ & Canaleja & Fernando y Tello & $? ?$ & DA\&C & Cardeña \\
\hline $\mathrm{C} 74$ & $01 / 05$ & Esgueva & Alfonso Sindini \& Eilo & $X$ & $\mathrm{D}$ & Cardeña \\
\hline $\mathrm{C} 75$ & $07 / 05$ & Cardeńadijo & Falconio & IV & DA\&C & Cardeña \\
\hline $\mathrm{C} 76$ & $31 / 05$ & Cardeñadijo & Asur & IV & $\mathrm{DA} \& \mathrm{C}$ & Cardeña \\
\hline $\mathrm{C} 77$ & $01 / 06$ & Cardeńadijo & Rapinato & IV & $\mathrm{DA} \& \mathrm{C}$ & Cardeña \\
\hline $\mathrm{C} 78$ & $27 / 06$ & Villalbilla & Urraca e hija Monina & $\mathrm{M} \& \mathrm{H}$ & DPf & Villalbilla \\
\hline $\mathrm{C} 79$ & $16 / 09$ & Barrio Eras & Egila & IV & V & Félix (P) \\
\hline $\mathrm{C} 80$ & $01 / 11$ & Villalbilla & Belaza & IV & $\mathrm{DA} \& \mathrm{C}$ & Villabilla \\
\hline S94f & $13 / 02$ & Melgar FC & Sendredo \& Albaro \& dońa Bona & $? ?$ & $\mathrm{~V}$ & Iscam \\
\hline$\$ 94 \mathrm{~g}$ & $23 / 01$ & Melgar FC & Zuleiman \& Hannoria & $\mathrm{X}$ & V & Iscam \\
\hline S94i & $17 / 06$ & Melgar FC & Abuzehar & IV & V & Iscam \\
\hline S94j & $23 / 04$ & Melgar FC & Susana \& 4 hijos & $\mathrm{M} \& \mathrm{H}$ & V & Iscam \\
\hline S94k & $24 / 04$ & Melgar FC & Osebio \& Gotina & $X$ & V & Iscam \\
\hline S941 & $28 / 04$ & Melgar FC & Teuda & I? & V & Iscam \\
\hline$S 120$ & ??/?? & \begin{tabular}{|l} 
Cansoles \\
\end{tabular} & Lecinio \& Sendino & $\mathrm{Hh}$ & V & Sahagún \\
\hline$\$ 121$ & $18 / 01$ & Villasoto & Claudia (viuda de Citi?) & IM & V & Melic (P) \\
\hline S122 & $07 / 03$ & Villa Helias & Frunimius \& Eilo & $\mathrm{X}$ & V & Sahagún \\
\hline$\$ 124$ & $27 / 03$ & Val Lorenzo & Vigila \& Deorosa & $\mathrm{X}$ & V & Muño Flaínez \& Froileuva \\
\hline S125 & $14 / 04$ & Porma & Guntina \& 4 hijos & $\mathrm{M} \& \mathrm{H}$ & V & Melic (P) \\
\hline S127a & $09 / 05$ & Villa Eiza & Vermudo & IV & V & Vicente (A) \\
\hline S127b & $09 / 05$ & Villa Eiza & Diaco & IV & V & Vicente (A) \\
\hline S128 & $10 / 05$ & Villar & Diego \& Leocadia & $\mathrm{X}$ & $\mathrm{D}$ & Sahagún \\
\hline L210 & $10 / 02$ & León & Aliporce \& Dońa Larga & $X$ & V & Citayo (P) \\
\hline L211 & $20 / 02$ & Montanelle & Hatita \& Leocadia & $X$ & V & Julián (P) \\
\hline L212 & $20 / 02$ & León? & Nobilia \& 5 hijos & $\mathrm{M} \& \mathrm{H}$ & V & Julián (P) \\
\hline L213 & $05 / 03$ & Talecias & Braolio \& Amelio & ?? & V & Dońa Paderna \\
\hline L214 & $12 / 03$ & León? & Armentario \& Mater & $\mathrm{X}$ & V & Obispo de León \\
\hline
\end{tabular}


EL GRAN INCENDIO CASTELLANO DE 949. HUELLA DIPLOMÁTICA

Y MEMORIA HISTÓRICA DE UN DESASTRE NATURAL

DAVID PETERSON

\begin{tabular}{|c|c|c|c|c|c|c|}
\hline Doc & DATE & NúCleo & ENAJENA & GRUPO & Tipo & AdQUIERE \\
\hline L215 & $29 / 03$ & Abellar & Pinniolus \& Cita & $\mathrm{X}$ & V & Abellar \\
\hline L216 & $31 / 03$ & Villobera & Valencia \& 9 hijos & $\mathrm{M} \& \mathrm{H}$ & V & Abellar \\
\hline $\mathrm{L} 217$ & $22 / 04$ & Paradilla & Vermudo \& hijos & $\mathrm{P \& H}$ & $\mathrm{V}$ & Obispo de León \\
\hline L218 & $01 / 05$ & Villa Abeiza & Mahepe \& Donna Kara & $\mathrm{X}$ & $\mathrm{V}$ & Fortunio \\
\hline L219 & $05 / 03$ & León & Julián \& Godica & $\mathrm{X}$ & $\mathrm{V}$ & Citayo $(\mathrm{P})$ \\
\hline L221 & $29 / 05$ & Río Cea & Abbaz \& Iaquinti & ¿? & $\mathrm{V}$ & Iquila (A) \\
\hline L222 & $17 / 05$ & Torre & Gesmira & IM & $\mathrm{D}$ & Flaino \& Brunildi \\
\hline L223 & $26 / 06$ & Abolereo & Mirantia \& Gigelo & $\mathrm{X}$ & $\mathrm{D}$ & Froila \& Gontrodo \\
\hline L224 & $28 / 06$ & Abellar & Gundisalva \& hijos & $\mathrm{M} \& \mathrm{H}$ & $\mathrm{V}$ & Abellar \\
\hline L225 & $19 / 07$ & Abellar & Matre, Maiscito \& Sisebuto & $\mathrm{Hh}$ & V & Abellar \\
\hline L226 & $22 / 08$ & Oteros & Todolfo \& Maria & $\mathrm{X}$ & $\mathrm{V}$ & Agube \& Auria \\
\hline L227 & $23 / 08$ & Ardón & Fortunio \& María & $\mathrm{X}$ & V & Valdevimbre \\
\hline L228 & $23 / 08$ & Rozola & Fredisilio & IV & V & Julián (P) \\
\hline L229 & $26 / 12$ & Esla & Artemio \& Liuvilo & $X$ & V & San Miguel \\
\hline L230 & ??!?? & León & Eulalia (enferma) & IM & D & Abellar \\
\hline
\end{tabular}

Leyenda:

- En sombra las transacciones del segundo semestre de 950.

- Tipo: $\mathrm{V}$ = venta; $\mathrm{D}=$ donación; $\mathrm{DPf}=$ donación por profiliación; $\mathrm{DA} \& \mathrm{C}=$ donación anima et corpora.

- Grupo: IM = individuo mujer; IV = individuo varón; $\mathrm{X}=$ matrimonio; $\mathrm{M} \& \mathrm{H}=$ madre e hijos; $\mathrm{P} \& \mathrm{H}=$ padre e hijos; $\mathrm{Hh}=$ hermanos.

- Otros: $\mathrm{P}=$ presbítero; $\mathrm{A}=$ abad. 